\title{
Assessment of Plasma Neurofilament Light as a Biomarker of Neuronal Injury in Young Adults with Perinatal HIV Infection
}

Beatriz Ruiz-Saez ( $\square$ bruizsaez@gmail.com )

Instituto de Investigación Sanitaria Gregorio Marañón (lisGM)

Manuela Martín-Bejarano

Hospital Universitario 12 De Octubre

Ana Martínez Aragon

Hospital Universitario 12 De Octubre

Magnus Gisslen

the Sahlgrenska Academy at the University of Gothenburg

Henrik Zetterberg

the Sahlgrenska Academy at the University of Gothenburg

Kaj Blennow

the Sahlgrenska Academy at the University of Gothenburg

Santiago Jiménez de Ory

Hospital Universitario Gregorio Marañon. Madrid

Susana Alvarez-Losada

Universidad Francisco de Vitoria

$M^{a}$ Angeles Muñoz-Fernández

Instituto de Investigación Sanitaria Gregorio Marañón (lisGM)

Helena Melero

Universidad Complutense de Madrid

Jose Tomás Ramos

Hospital Universitario Clínico San Carlos

Maria Luisa Navarro

Hospital Universitario Gregorio Marañon. Madrid

Maria Isabel González-Tomé

Hospital Universitario 12 De Octubre

\section{Research Article}

Keywords: Plasma NfL, perinatal HIV, neuroimaging, neurocognitive, CART 
Posted Date: August 17th, 2021

DOl: https://doi.org/10.21203/rs.3.rs-787438/v1

License: (c) (i) This work is licensed under a Creative Commons Attribution 4.0 International License. Read Full License 


\section{Abstract}

Background: Higher plasma concentration of neurofilament light ( $\mathrm{pNfL}$ ) is associated with neurodegeneration. However, to our knowledge, up to now, there are no data in HIV patients with infection due to vertical transmission. This is the first study to report pNfL in a cohort of HIV perinatally infected (PHIV) young adults compared with non-HIV (HIV-) controls.

Methods: Thirty-three PHIV patients and 25 age-matched HIV- were recruited to this cross-sectional study. Plasma NfL concentrations were compared between both groups. In a subgroup of 48 participants (25 PHIV patients and 23 HIV-), brain volumes through magnetic resonance imaging (MRI) and neuropsychological testing (NT), were also conducted and compared with pNfL values.

Plasma NfL concentration was measured using Single Molecule Array (Simoa) immunoassay.

NT included fluid intelligence and processing speed through the WAIS-IV Coding subtest, and the Stroop Test.

\section{Results}

Fifty-eight participants were included, median age 20.7 years [IQR 17.8-23.4]. $100 \%$ of the patients were under antiretroviral treatment (cART) and $85 \%$ had viral load $<50$ copies $/ \mathrm{ml}$.

Although no statistically significant differences were found between patients and controls regarding pNfL concentration, there was a trend towards higher levels in patients with viral load $>50$ copies $/ \mathrm{ml}$.

With regard to brain volumes and NT, in the PHIV group, lower white matter volumes and lower score in the coding subtest were associated with higher $\mathrm{pNfL}$ values.

\section{Conclusions}

Most PHIV adolescents under CART have similar levels of pNfL than HIV-. As reported in adults, those with HIV-RNA >50 copies/ml showed higher values and lower white matter volumes that may imply an ongoing CNS injury. Plasma NfL could be a feasible biomarker of CNS injury in PHIV patients with unsuppressed viral load.

\section{Background}

The incidence of HIV encephalopathy and severe neurological complications has been significantly reduced in perinatally HIV-infected patient since the introduction of combined antiretroviral therapy ${ }^{1}$. Nevertheless, in the PHIV population, CNS invasion of HIV occurs within the first 3 weeks of life, with a subsequent immune activation throughout the primary infection. This is among the most important reasons why research concerning the detection of persistent neurologic problems is essential ${ }^{2}$. This 
research could explain why less severe cognitive impairment often could persists in this population, especially in children who did not start treatment in early life ${ }^{3}$.

To better understand how the development of brain injury and intrathecal immune activation and inflammation occur, several CSF biomarkers have been investigated, with CSF neurofilament light (NfL) being the most useful biomarker for the study of HIV-induced neuroaxonal injury ${ }^{4}$. This major structural component of myelinated axons is essential to maintain axonal calibre and to facilitate effective nerve conduction ${ }^{5}$. It is a sensitive, but disease-unspecific, biomarker for neuronal degeneration or acute neuronal damage ${ }^{6}$.

Several studies performed in HIV-infected adults have shown increased CSF NfL levels in patients with HIV associated dementia but also in neuroasymptomatic subjects with low CD $4+$ T-cell counts ${ }^{5-7}$. Meanwhile, treated and virologically suppressed people living with HIV have lower CSF NfL levels, but this is still slightly higher than HIV-negative individuals ${ }^{5}$.

Measuring CSF NfL uses the invasive procedure of lumbar puncture, so its use is limited. Therefore, a new technique has been developed using ultrasensitive (Simoa) immunoassay for measuring NfL in blood samples ${ }^{8}$. Results derived using this new method show that plasma NfL correlates strongly with CSF NfL levels at all stages of HIV infection ${ }^{8}$.

To our knowledge, no study of plasma or CSF NfL has been performed in the perinatally HIV-infected population.

It is worth stating, that in recent years, several neuroimaging studies have been performed in PHIV children and young adults demonstrating that, even in the CART era, there are alterations and lower volumes in brain structure ${ }^{9}$. As NfL CSF levels reflect leakage from injured or degenerating neurons, it correlates with white matter lesions and other injuries to subcortical brain regions ${ }^{10}$. To date, there are no studies correlating white matter brain volumes and $\mathrm{pNfL}$ values in perinatally HIV-infected patients.

Therefore, the current study aimed to investigate, firstly, the pNfL levels in a group of PHIV population and compared them with a group of HIV-negative controls participating in the NeuroCoRISpe study. Secondly, a sub-study was performed to explore possible correlations between $\mathrm{pNfL}$ concentration and white matter volumes and processing speed performance in a group of participants.

\section{Methods}

\section{Population and Study Design}

A multicentre cross-sectional study was carried out from 2016 to 2018 in a cohort of vertically HIVinfected adolescents and young adults followed at five public hospitals participating in the Madrid Cohort 
of HIV-Infected Children and Adolescents and in the Cohort of Spanish Pediatric HIV Network (CoRISpe) 11

The HIV- were selected from voluntary recruitment through advertising.

Thirty-three PHIV patients and 25 HIV-controls matched by age were recruited.

For the study, all participants met the following inclusion criteria: (1) age 15 to 25 years old, (2) absence of neurological or psychiatric disorder other than history of older HIV encephalopathy, (3) HIV participants should be under CART treatment.

Participants with current brain infection, neurological or psychiatric disorder, those who referred history of drug or alcohol abuse, or had any congenital abnormality, were excluded.

The Institutional Review Boards (IRBs) of each research centre approved the study and written informed consent was obtained from all participants. Where participants were underage, an assent form was signed by themselves, with legal guardians providing the informed consent in accordance with the Helsinki Declaration.

\section{HIV-related measures}

In relation to the control of the infection the next parameters were collected: CDC classification, encephalopathy, suppressed viral load (defined as plasma HIV-RNA $<50$ copies/ml), time of suppressed viral load, viral load in detectable patients, total numbers, and percentages of CD4 nadir, and current CD4, CD4 / CD8 ratio, CART history and adherence to treatment. These data were collected from clinical charts and the CoRISpe database.

\section{Plasma NfL measurements}

Whole blood was collected in EDTA tubes which were sent to the Spanish HIV HGM BioBank for centrifugation $\left(2000 \mathrm{~g}\right.$ ) and aliquoted into cryo tubes in $1 \mathrm{~mL}$ portions and stored at $-80^{\circ}$ for subsequent analysis ${ }^{12}$. Plasma NfL concentration was measured using a sensitive in-house sandwich immunoassay on the (Simoa) HD-1 Analyzer (Quanterix, Billerica, MA), as previously described in detail ${ }^{8}$.

\section{Neuropsychological and neuroimaging sub-study}

A subgroup of 48 participants (25 PHIV +, 23 HIV-) with no differences in sex, age, level of education and socioeconomic status between groups, underwent NT testing and MRI scan. These subjects participated previously in a neurocognitive and neuroimaging study (Ruiz-Saez et al, 2021) ${ }^{13}$ and whole blood was collected at the same time and stored at the HIV HGM Biobank for subsequent pNfL analysis.

The NT included fluid intelligence (FI) by the Kaufman Brief Intelligence Test ${ }^{14}$ (K-BIT; Kaufman \& Kaufman, 2000), and processing speed measured through two tests, the Digit Symbol-Coding subtest of 
the Wechsler Adult Intelligence Scale- 4 th edition $^{15}$ (WAIS-IV, Weschler, 2012), and the first trial of the Stroop Test ${ }^{16}$ (Golden, 2001).

In this study, we focused on fluid intelligence to make sure abstract reasoning and problem solving in novel situations independently of experience was average in both groups. Processing speed was also evaluated, because is one of the main cognitive deficits in HIV patients ${ }^{17}$. Scores on all neuropsychological tests were converted into a Z-score relative to HCs. Scores on the Digit SymbolCoding and Stroop-Card 1 were averaged into one PS composite Z-score.

\section{MRI data acquisition}

Different MRI scanner systems were used at each hospital study site. For specific details of the acquisition parameters see Supplementary material.

Image quality was assessed in two independent processes. Radiologist checked for the presence of any brain pathology, such as tumour, cyst, or any other lesion.

In addition, image quality and processing experts checked for motion artefacts, low contrasts, incomplete whole brain coverage, low SNR and low resolution. In a further analysis, all the acquisitions were correlated to determine the homogeneity of the image sample.

\section{Image processing}

The standard processing pipeline for volume based morphometry provided by The Computational Anatomy Toolbox (CAT12, http://dbm.neuro.uni-jena.de/cat/ version 1492), as an extension of SPM (https://www.fil.ion.ucl.ac.uk/spm/software/spm12/ version 7487), was used for tissue segmentation and the extraction of tissue volumes. To measure regional brain volumes, native segmented images were parceled in regions of interest (ROI) according to the Hammers atlas ${ }^{18-19}$ (Hammers et al. 2003; Gousias et al. 2008) and tissue volumes $\left(\mathrm{mm}^{3}\right)$ were estimated for each ROI and normalized by the total intracranial volume for each subject.

\section{Statistical analysis}

Categorical variables were summarized by using counts and proportions and continuous variables employing medians and interquartile ranges (IQR) or means and standard deviations (SD). Comparisons between patients and controls (NfL, age, Fluid Intelligence, Stroop, Coding and Composite z-score) were performed with the Student $t$ test or the Mann-Whitney $U$ test, if the variables did not follow a normal distribution. Comparisons between categorical variables were assessed using the Chi-square or the Fisher test. In the case of patients with and without undetectable viral load and controls, variables were analyzed with the Kruskal-Wallis test. Regarding the PHIV group, univariate analysis was performed to study associations between HIV variables and NfL. Spearman's correlation test was used to assess association $\mathrm{pNfL}$ concentrations and white matter volumes. $P$ values less than 0.05 were considered statistically significant. All analyses were performed using SPSS software ver. 22.0 (IBM, Armonk, NY, USA). Figure 2 was made using Stata Version 12 (STATA Corp, Texas, USA). 


\section{Results}

Thirty-three young adults with perinatal HIV infection and 25 HIV-negative individuals were included.

In the PHIV group, 54\% were women and $73 \%$ were Caucasians. Median age was 20.7 years (IQR $17.8-$ 23.4). In the HIV-negative group, median age was 21.3 years (IQR 19.7-23.1) and 60\% were women.

There were no significant differences between the two groups regarding these characteristics $(p>0.05)$.

Regarding the PHIV group, $42 \%$ had a history of previous AIDS-defining diagnoses ( $21 \%$ with old and stable encephalopathy). At assessment, $100 \%$ were under cART for a median time of 16.42 years (IQR 12.99-18.70), and $85 \%$ had suppressed viral load (HIV RNA $<50$ copies/ml); Only five patients had HIVRNA > 50 copies/ml with a median of 69900 copies/ml (IQR 36774-267541). Those five patients had detectable viral load for a median of 5.75 years (IQR 5.03-16.75). Median CD4 was 738 cells $/ \mathrm{mm}^{3}$ (IQR 578-978) and median CD4 nadir 274 (IQR 104-382). (Table 1).

\section{Table 1}

Clinical measures in 33 PHIV patients ( $\mathrm{n}(\%)$ or median [IQR]) 


\begin{tabular}{|l|l|}
\hline $\begin{array}{l}\text { Age at HIV diagnosis } \\
\text { (years) }\end{array}$ & $0.50[0.24-4.08]$ \\
\hline CDC Stage C3 & $14(42.4 \%)$ \\
\hline Encephalopathy & $7(21.2 \%)$ \\
\hline NADIR CD4 (cells/mm3) & $274[124-376]$ \\
\hline NADIR CD4 (\%) & $12[6-17]$ \\
\hline CD4 count (cells/mm3) & $718[490-771]$ \\
\hline CD4 count (\%) & $36[32-39]$ \\
\hline CD4/CD8 & $1.03[0.80-1.31]$ \\
\hline $\begin{array}{l}\text { Age at treatment onset } \\
\text { (years) }\end{array}$ & $1.33[0.44-4.56]$ \\
\hline $\begin{array}{l}\text { Age at onset on cART } \\
\text { (years) }\end{array}$ & $4.28[1.03-6.66]$ \\
\hline $\begin{array}{l}\text { Total time of treatment } \\
\text { with cART (years) }\end{array}$ & $16.42[12.99-18.70]$ \\
\hline Patients with uVL & $28(85 \%)$ \\
\hline Time with uVL (years) & $9.97[6.92-13,48]$ \\
\hline $\begin{array}{l}\text { VL in detectable patients } \\
\text { (cop/ml) (N = 5) }\end{array}$ & $69900[36774-$ \\
\hline
\end{tabular}

No statistically significant differences were found between patients and controls regarding $\mathrm{PNfL}$ concentrations, but higher levels of pNfL were found in patients with increased viral load compared compared with undetectable patients and controls with a media pNfL of $9.19 \mathrm{pg} / \mathrm{ml}$ (SD 5.18) in patients with detectable viral load vs $6.6 \mathrm{pg} / \mathrm{ml}$ (SD 4.15) in undetectable patients and $5.29 \mathrm{pg} / \mathrm{ml}$ (SD 1.75) in the control group $(p=0.059)$ (Fig. 1).

Furthermore, no correlations were found between $\mathrm{pNfL}$ levels and viral load, time to diagnosis, time on CART, CDC stage or presence of encephalopathy ( $p>0.05$ for all comparisons). 
In the correlation sub-study of pNfL with neuroimaging and neuropsychological evaluations, $25 \mathrm{PHIV}+$ and 23 HIV-negative controls were evaluated. Sociodemographic characteristics are described in Table 2. In relation to NT, we found that PHIV had significantly lower Fl, but both groups had average results. Differences between groups in Stroop test performance were observed, but not in Coding. The mean PS composite z-score was lower in the PHIV group (Mean Z-score - 0.68 (SD 0.98)) compared to the HIVnegative group (Mean Z-score $0.00($ SD 1.00)) $(p<0.05)$

Table 2

Means (standard deviations) of demographic, psychosocial, neurocognitive and psychopathological characteristics.

\begin{tabular}{|c|c|c|c|}
\hline & PHIV (n = 25) & HIV- $(n=23)$ & $P$ value \\
\hline Sex (female) $(\%, n)$ & $64(16)$ & $56,5(13)$ & 0.597 \\
\hline Age at assessment & $21.0(3.03)$ & $20.9(2.66)$ & 0.522 \\
\hline Level of education $(\%, n)$ & $N=24$ & $N=21$ & 0.146 \\
\hline Low & $62.5(15)$ & $76.2(16)$ & \\
\hline Medium & $16.7(4)$ & $0(0)$ & \\
\hline High & $20.8(5)$ & $23.8(5)$ & \\
\hline Annual Income $(\%, n)$ & $N=23$ & $N=22$ & 0.135 \\
\hline Low & $43.5(10)$ & $59.1(13)$ & \\
\hline Medium & $30.4(7)$ & $36.4(8)$ & \\
\hline High & $26.1(6)$ & $4.5(1)$ & \\
\hline Caucasian $(\%, n)$ & $72(18)$ & $56,5(13)$ & 0.263 \\
\hline Fluid Intelligence * & $-0.72(1.13)$ & $0.01(0.98)$ & 0.021 \\
\hline Stroop & $-0.84(1.09)$ & $0.00(0.99)$ & 0.009 \\
\hline Coding & $-0.31(0.86)$ & $-0.12(1.14)$ & 0.512 \\
\hline Composite Processing Speed Z-score & $-0.68(0.98)$ & $-0.00(1.00)$ & 0.025 \\
\hline
\end{tabular}

Regarding brain volumes, the HIV infected group had significantly lower regional white matter volumes in left and right cerebellum $(p=0.030, p=0.028)$, lateral occipital lobe $(p=0.020)$, left and right nucleus accumbens $(p=0.010, p<0.001)$,

left and right occipital lobe $(p=0.020, p=0.042)$ and left postcentral gyrus $(p=0.022)$, but no significant differences were found in total white matter volumes. 
In the HIV group, Spearman's correlation test revealed negative association between pNfL concentrations and different regional white matter volumes of left and right cerebellum $(r-, 440 p=0.028 ; r-0386, p=$ 0.056), left and right brainstem $(r-440 p=0.028 ; r-, 417 p=0.038)$ and right nucleus accumbens $(r-403$, $p=0.046)$ Fig. (2), and also a negative correlation was found between $\mathrm{pNfL}$ concentration and Coding score $(r-425 p=0.039)$. This association between $\mathrm{NfL}$ and brain volumes and coding score persisted when controlling for undetectable viral load.

\section{Discussion}

$\mathrm{NfL}$ is a neurofilament subunit particularly abundant in axons ${ }^{20}$. Plasma NfL concentration was recently reported as a potential prognostic biomarker of disease onset and progression in neurodegenerative diseases including $\mathrm{HIV}^{4-8}$.

In this study, we have shown that treated and virologically suppressed PHIV people, presented pNfL concentrations similar of those found in HIV-negative individuals. In addition, even considering the limitation of the small sample, patients with detectable viral load had higher pNfL levels, showing that persistent viral replication may contribute to neuronal damage.

This has been demonstrated similarly in the HIV adult population, in which the HIV-driven axonal degeneration can be halted by CART, which correlates to reduced CSF and pNfL concentrations over time after CART initiation ${ }^{5,21-22}$. These results emphasize the importance of an early and continuous antiretroviral therapy to avoid neuronal damage in children.

In this exploratory study of PHIV adolescents and young adults, we found that higher pNfL concentration was significantly associated with lower regional brain volume and lower coding score. Similarly, Anderson et al. published that a higher pNfL was significantly associated with worse neuropsychological performance in the HIV adult population ${ }^{23}$.

High concentration of $\mathrm{NfL}$ has been shown in multiple neurological diseases where processing speed is also one of the most affected cognitive processes, such as amyotrophic lateral sclerosis (ALS) 24,25 (Menke et al., 2015; Lu et al., 2015), Alzheimer's disease ${ }^{26}$ (Mattsson et al., 2017) and frontotemporal dementia ${ }^{27}$ (Rohrer et al., 2016). Processing speed performance is considered to depend to a large extent on the properties of the white matter ${ }^{28-30}$ (Posthuma et al, 2003; Borghesani et al., 2013; Jacobs et al., 2013). White matter includes myelinated axons in the brain, and the thickness of the myelin sheath is associated with nerve conduction velocity; therefore, its relation to processing speed and $\mathrm{NfL}$ seems consistent. Hence plasma NfL could be a feasible biomarker of milder neurocognitive alterations in the PHIV population.

Likewise, increased NfL levels and reduced brain volume in cortical and subcortical grey matter and within the white matter has been found in patients with different neurodegenerative conditions ${ }^{31-32}$. 
However, research performed in the HIV adult population found that CSF neuronal damage biomarkers, including NfL, were not associated with imaging measures of brain structures ${ }^{33}$.

It should be noted that NfL has the limitation that it is not a disease-specific biomarker. As we have mentioned, elevated NfL is observed in many other neurological disorders, including neurodegenerative diseases, peripheral neuropathy, and traumatic brain injuries ${ }^{6,34}$.

Regarding brain volumes we found that the HIV group showed WM atrophy in selected brain regions despite being on CART for years. Some studies performed in adolescents living with PHIV has reported similar results showing lower white matter volumes when compared with HIV negative controls s $^{35,36}$.

Limitations of this study include the small sample size and lack of longitudinal biomarker data. The small sample size has been partially compensated for by strict selection criteria for the control group. Moreover, other limitations of the study are that this age group is potentially more likely to be involved in sports with head trauma, and this group of population may have different stressors, that have not been measured. Number of adverse childhood events (ACEs) would be a useful marker but has not been used. These are potentially important considerations in the adolescent age group during a time of dynamic myelination.

Finally, this current work was exploratory and therefore multiple statistical tests were performed, which might have resulted in type I errors.

Strengths of our study are, the inclusion of young adults living with PHIV, who have not previously been examined regarding plasma $\mathrm{NfL}$ levels, and that we were able to correlate brain volumes and processing speed in this population.

This research is representative of most young adults living with HIV vertically infected in developed healthcare systems. Moreover, thanks to the current great improvements in the diagnosis and treatment of HIV infection, this population that were born in the preTAR era making the study unique.

\section{Conclusions}

We can conclude that the ultrasensitive method to measure $\mathrm{pNfL}$ concentration provides an easily accessible biomarker in perinatally HIV infected patients avoiding lumbar puncture. Nevertheless, it remains unclear how pNfL varies in the PHIV population with virologic suppression or how its levels could be influenced in this population by the earlier initiation of effective antiretroviral therapy. Therefore, larger longitudinal studies are required in this group to further evaluate $\mathrm{pNfL}$ as a clinically useful biomarker of neurological deterioration.

\section{Declarations}

\section{Ethics approval and consent to participate:}


The Institutional Review Boards (IRBs) of each research centre approved the study and written informed consent was obtained from all participants. Where participants were underage, an assent form was signed by themselves, with legal guardians providing the informed consent in accordance with the Helsinki Declaration.

\section{Consent for publication:}

We hereby verify that the manuscript has not been submitted or accepted elsewhere. All authors have given consent for its publication.

\section{Availability of data and material:}

The authors confirm that all data underlying the findings are fully available without restriction. All relevant data are within the paper and its Supporting Information files.

\section{Competing interests:}

a) NeuroCoRISpe group declare no competing financial interest.

b) The Sahlgrenska Academy at the University of Gothenburg group have no

competing interests that could be construed as influencing the contents of this paper. However, the authors list the following general potential conflicts of interest:

HZ has served on scientific advisory boards for Denali, Roche Diagnostics, Wave, Samumed, Siemens Healthineers, Pinteon Therapeutics and CogRx, has given lectures in symposia sponsored by Fujirebio, Alzecure and Biogen, and is a co-founder of Brain Biomarker Solutions in Gothenburg AB (BBS), which is a part of the GU Ventures Incubator Program (outside submitted work).

KB has served as a consultant, at advisory boards, or at data monitoring committees for Abcam, Axon, Biogen, JOMDD/Shimadzu. Julius Clinical, Lilly, MagQu, Novartis, Roche Diagnostics, and Siemens Healthineers, and is a co-founder of Brain Biomarker Solutions in Gothenburg AB (BBS), which is a part of the GU Ventures Incubator Program.

MG reports personal fees (scientific advisory boards, lectures) from Gilead Sciences, GSK/ViiV, MSD, Biogen, Amgen, Novocure, Novo Nordic and research grants from Gilead Sciences, all outside the submitted work.

\section{Funding:}


a)_NeuroCoRispe group and Spanish HIV HGM BioBank:

This work was funded by the Instituto de Salud Carlos III, Acción Estratégica en Salud [FIS 15/00694] and Fondos FEDER of the UE. CoRISpe is integrated in the Spanish AIDS Research Network (RIS), supported by the Instituto de Salud Carlos III [RD16/0025/0017-ISCIII-FEDER,RD16/0025/0019-ISCIII-FEDER, RD16/0025/0024-ISCIII-FEDER and RIS -EPICLIN12/2012] ;BRS was funded by the Spanish Ministry of Science and Innovation- Instituto de Salud Carlos III (Contratos Rio Hortega CM 16/00224). This study takes part of the BRS PhD at Universidad de Alcalá (Madrid, Spain).

b)_Sahlgrenska Academy at the University of Gothenburg_group:

This work was supported by the Swedish state, under an agreement between the Swedish government and the county councils (ALF agreement ALFGBG-717531).

$\mathrm{HZ}$ is a Wallenberg Scholar supported by grants from the Swedish Research Council (\#2018-02532), the European Research Council (\#681712), Swedish State Support for Clinical Research (\#ALFGBG-720931), the Alzheimer Drug Discovery Foundation (ADDF), USA (\#201809-2016862), the AD Strategic Fund and the Alzheimer's Association (\#ADSF-21-831376-C, \#ADSF-21-831381-C and \#ADSF-21-831377-C), the Olav Thon Foundation, the Erling-Persson Family Foundation, Stiftelsen för Gamla Tjänarinnor, Hjärnfonden, Sweden (\#F02019-0228), the European Union's Horizon 2020 research and innovation programme under the Marie Skłodowska-Curie grant agreement No 860197 (MIRIADE), and the UK Dementia Research Institute at UCL.

KB is supported by the Swedish Research Council (\#2017-00915), the Alzheimer Drug Discovery Foundation (ADDF), USA (\#RDAPB-201809-2016615), the Swedish Alzheimer Foundation (\#AF-742881), Hjärnfonden, Sweden (\#F02017-0243), the Swedish state under the agreement between the Swedish government and the County Councils, the ALF-agreement (\#ALFGBG-715986), and European Union Joint Program for Neurodegenerative Disorders (JPND2019-466-236).

\section{Authors' contributions:}

BRS, MMB, MGT, MM conceptualized and designed the study, participated in analysis and interpretation of the data, drafted the initial manuscript and contributed and approved the final manuscript as submitted. BRS, MMB, MG, HZ, KB, AMA, SOJ, SAL, MAM, HM, JTR, MLN, MIGT were involved in the provision of study subjects, reviewed the manuscript drafts, and approved the final manuscript as submitted. All authors read and approved the final manuscript

\section{Acknowledgments}

This study would not have been possible without the collaboration of all the participants, medical and nursing staff, and data managers who have taken part in Neurocorispe Project of The Pediatric National 
AIDS Research Network of Spain (CORISPE). This work has been performed in collaboration with Biobank Hospital Gregorio Marañon (HGM) and the Sahlgrenska Academy at the University of Gothenburg, Gothenburg, Sweden

\section{References}

1. Patel $K$, Ming X, Williams PL, et al. Impact of HAART and CNS penetrating antiretroviral regimens on HIV encephalopathy among perinatally infected children and adolescents. AIDS. 2009;23:18931901.

2. Cohen S, Caan MWA, Mutsaerts H-J, Scherpbier HJ, Kuijpers TW, Reiss P, et al. Cerebral injury in perinatally HIV-infected children compared to matched healthy controls. Neurology 2016; 86:19-27

3. Judd A, Le Prevost M, Melvin D, et al. Cognitive Function in Young Persons With and Without Perinatal HIV in the AALPHI Cohort in England: Role of Non-HIV-Related Factors. CID 2016;63(10):1380-7

4. Yilmaz A, Blennow K, Hagberg L, et al. Neurofilament light chain protein as a marker of neuronal injury: review of its use in HIV-1 infection and reference values for HIV negative controls. Expert Rev Mol Diagn 2017; 17:761-70.

5. Jessen Krut J, Mellberg T, Price RW, et al. Biomarker evidence of axonal injury in neuroasymptomatic HIV-1 patients. Plos One. 2014;9(2):e88591.

6. Ashton NJ, Hye A, Rajkumar AP, Leuzy A, Snowden S, Suárez-Calvet M, Karikari T, Schöll M, La Joie R, Rabinovici GD, Höglund K, Ballard C, Hortobagyi T, Svenningsson P, Blennow K, Zetterberg H, Aarsland D. An update on blood-based biomarkers for non-Alzheimer neurodegenerative disorders. Nat Rev Neurol 2020;16(5):265-284.

7. Peterson J, Gisslen M, Zetterberg H, et al. Cerebrospinal fluid (CSF) neuronal biomarkers across the spectrum of HIV infection: hierarchy of injury and detection. Plos One. 2014;9(12):e116081.

8. Gisslen M, Price RW, Andreasson U, et al. Plasma concentration of the neurofilament light protein (NFL) is a biomarker of CNS injury in HIV infection: a cross-sectional study. EBioMedicine. 2016;3:135-140.

9. Hoare, Jacqueline, Jean-Paul Fouche, Nicole Phillips, John A. Joska, Landon Myer, Heather J. Zar, y Dan J. Stein. «Structural Brain Changes in Perinatally HIV Infected Young Adolescents in South Africa»: AIDS, 2018, 1. https://doi.org/10.1097/QAD.0000000000002024.

10. Jonsson $M$, Zetterberg $H$, van Straaten $E$, et al. Cerebrospinal fluid biomarkers of white matter lesions - cross-sectional results from the LADIS study. Eur J Neurol. 2010 Mar;17(3):377-82

11. de Jose MI, Jiménez de Ory S, Espiau M, Fortuny C, Navarro ML, Soler-Palacín P, Muñoz-Fernandez MA, working groups of CoRISpe and HIV HGM BioBank. A new tool for the paediatric HIV research: general data from the Cohort of the Spanish Paediatric HIV Network (CoRISpe). BMC Infect Dis 2013 Jan 2;13:2. doi: 10.1186/1471-2334-13-2. 
12. García-Merino I, de Las Cuevas N, Jiménez JL, et al. Pediatric HIV BioBank: a new role of the Spanish HIV BioBank in pediatric HIV research. AIDS Res Hum Retroviruses. 2010;26:241-244.

13. Ruiz-Saez B, García MM, de Aragon AM, Gil-Correa M, Melero H, Malpica NA, de Ory SJ, Zamora B, Guillen S, Rojo P, Falcon-Neyra L, Alvarez A, Fernandez P, Lorente-Jareño ML, Ramos JT, Sainz T, Velo C, Navarro ML, Gonzalez-Tomé MI; Cohorte Nacional de VIH pediátrica de la RED RIS (CoRISpe), Madrid, Spain. Effects of perinatal HIV-infection on the cortical thickness and subcortical gray matter volumes in young adulthood. Medicine (Baltimore). 2021;16:100-115.

14. Kaufman, A. S., Kaufman, N. L. (2000) Manual del Test breve de inteligencia de Kaufman (K-BIT) [Manual for the Kaufman Brief Intelligence Test]. Madrid, Spain: TEA Ediciones.

15. Wechsler, D. (2012). WAIS-IV. Escala de inteligencia de Wechsler para adultos-IV. Manual de aplicación y corrección. Madrid: NCS Pearson, Inc. Edición original, 2008.

16. Golden, C. J. (2020). STROOP. Test de Colores y Palabras - Edición Revisada (B. Ruiz-Fernández, T. Luque y F. Sánchez-Sánchez, adaptadores). Madrid: TEA Ediciones.

17. Phillips N, Amos T, Kuo C, Hoare J, Ipser J, Thomas KG, Stein DJ. HIV-Associated Cognitive Impairment in Perinatally Infected Children: A Meta-analysis. Pediatrics. 2016 Nov;138(5):e20160893

18. Hammers A, Allom R, Koepp MJ, Free SL, Myers R, Lemieux L, Mitchell TN, Brooks DJ, Duncan JS. Three-dimensional maximum probability atlas of the human brain, with particular reference to the temporal lobe. Hum Brain Mapp 2003, 19: 224-247.

19. Gousias IS, Rueckert D, Heckemann RA, Dyet LE, Boardman JP, Edwards AD, Hammers A. Automatic segmentation of brain MRIs of 2-year-olds into 83 regions of interest. Neuroimage 2008 Apr 1;40(2):672-684.

20. Lorenzo Gaetani, Kaj Blennow, Paolo Calabresi, Massimiliano Di Filippo, Lucilla Parnetti, Henrik Zetterberg. gNeurofilament light chain as a biomarker in neurological disorders J Neurol Neurosurg Psychiatry. 2019 Aug;90(8):870-881. doi: 10.1136/jnnp-2018-320106. Epub 2019 Apr 9.

21. Mellgren A, Price RW, Hagberg L, Rosengren L, Brew BJ, et al. (2007) Antiretroviral treatment reduces increased CSF neurofilament protein (NfL) in HIV-1 infection. Neurology 69: 1536-1541.

22. Price RW, Peterson J, Fuchs D, Angel TE, Zetterberg H, et al. (2013) Approach to cerebrospinal fluid (CSF) biomarker discovery and evaluation in HIV infection. J Neuroimmune Pharmacol 8: 11471158.

23. Anderson A, Easley KA, Kasher N. Neurofilament light chain in blood is negatively associated with neuropsychological performance in HIV-infected adults and declines with initiation of antiretroviral therapy. Journal of NeuroVirology (2018) 24:695-701

24. Menke RA, Gray E, Lu CH, Kuhle J, Talbot K, Malaspina A, et al. CSF neurofilament light chain reflects corticospinal tract degeneration in ALS. Ann Clin Transl Neurol. 2015 Jul;2(7):748-55

25. Lu CH, Macdonald-Wallis C, Gray E, Pearce N, Petzold A, Norgren N, et al. Neurofilament light chain: a prognostic biomarker in amyotrophic lateral sclerosis. Neurology 2015;84:2247-2257.

26. Mattsson N, Andreasson U, Zetterberg H, Blennow K; Alzheimer's Disease Neuroimaging Initiative. Association of plasma neurofilament light with neurodegeneration in patients with alzheimer 
disease. JAMA Neurol 2017;74:557-566.

27. Rohrer JD, Woollacott IO, Dick KM, Brotherhood E, Gordon E, Fellows A, et al. Serum neurofilament light chain protein is a measure of disease intensity in frontotemporal dementia. Neurology. 2016 Sep 27;87(13):1329-36

28. Posthuma D, Baaré WFC, Hulshoff Pol HE, Kahn RS, Boomsma DI, De Geus EJC. Genetic correlations between brain volumes and the WAIS-III dimensions of verbal comprehension, working memory, perceptual organization, and processing speed. Twin Research. 2003;6(02):131-9.

29. Borghesani PR, Madhyastha TM, Aylward EH, Reiter MA, Swarny BR, Schaie KW, et al. The association between higher order abilities, processing speed, and age are variably mediated by white matter integrity during typical aging. Neuropsychologia. 2013;51(8):1435-44. pmid:23507612

30. Jacobs HIL, Leritz EC, Williams VJ, Van Boxtel MPJ, Elst Wvd, Jolles J, et al. Association between white matter microstructure, executive functions, and processing speed in older adults: the impact of vascular health. Human brain mapping. 2013;34(1):77-95. pmid:21954054

31. Eileanoir B Johnson ${ }^{1}$, Lauren M Byrne ${ }^{1}$, Sarah Gregory ${ }^{1}$, Filipe B Rodrigues ${ }^{1}$, Kaj Blennow ${ }^{1}$, Alexandra Durr ${ }^{1}$, Blair R Leavitt ${ }^{1}$, Raymund A Roos ${ }^{1}$, Henrik Zetterberg ${ }^{1}$, Sarah J Tabrizi ${ }^{1}$, Rachael I Scahill ${ }^{1}$, Edward J Wild ${ }^{2}$, TRACK-HD Study Group. Neurofilament light protein in blood predicts regional atrophy in Huntington disease. Neurology. 2018 Feb 20;90(8):e717-e723.

32. Li QF, Dong Y, Yang L, Xie JJ, Ma Y, Du YC, Cheng HL, Ni W, Wu ZY. Neurofilament light chain is a promising serum biomarker in spinocerebellar ataxia type 3. Mol Neurodegener. 2019 Nov 4;14(1):39.

33. Van Zoest R, Underwood J, De Francesco D, Sabin CA, Cole JH, Wit FW, et al. Structural Brain Abnormalities in Successfully Treated HIV Infection: Associations With Disease and Cerebrospinal Fluid Biomarkers. J Infect Dis 2017 Dec 27;217(1):69-81 doi: 10.1093/infdis/jix553.

34. Bridel C, van Wieringen WN, Zetterberg H, Tijms BM, Teunissen CE, Alvarez-Cermeno JC, et al. Diagnostic value of cerebrospinal fluid Neurofilament light protein in neurology: a systematic review and meta-analysis. JAMA Neurol. 2019; Epub ahead of print.

35. Cohen S, Caan MWA, Mutsaerts H-J, Scherpbier HJ, Kuijpers TW, Reiss P, et al. Cerebral injury in perinatally HIV-infected children compared to matched healthy controls. Neurology 2016; 86:19-27

36. Sarma, M. K., Nagarajan, R., Keller, M. A., Kumar, R., Nielsen-Saines, K., Michalik, D. E., et al (2014). Regional brain gray and white matter changes in perinatally HIV-infected adolescents. Neurolmage: Clinical, 4, 29-34

\section{Figures}




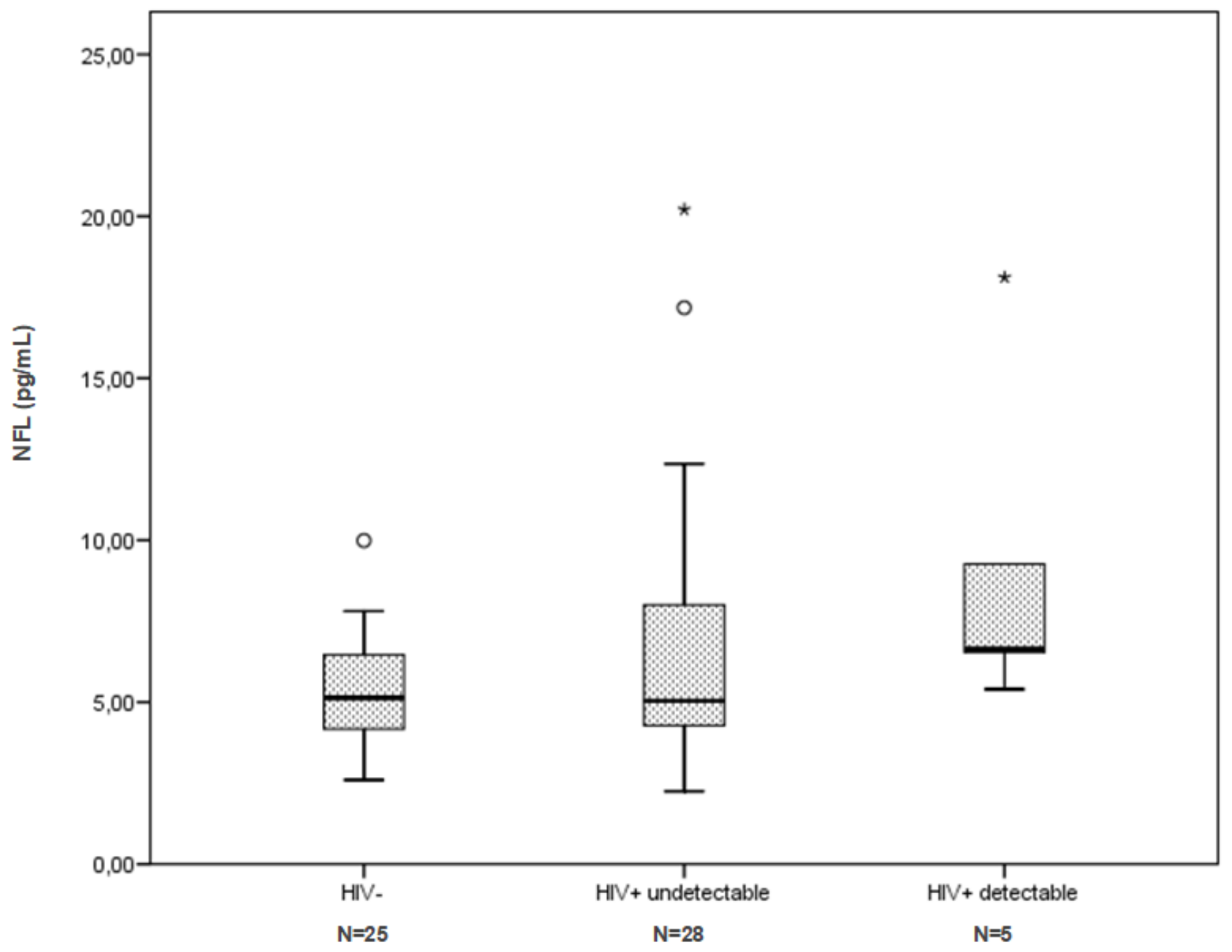

Figure 1

Plasma NfL concentrations in 3 different groups: HIV-negative control group, PHIV patients with detectable VL, and PHIV patients with undetectable viral load 

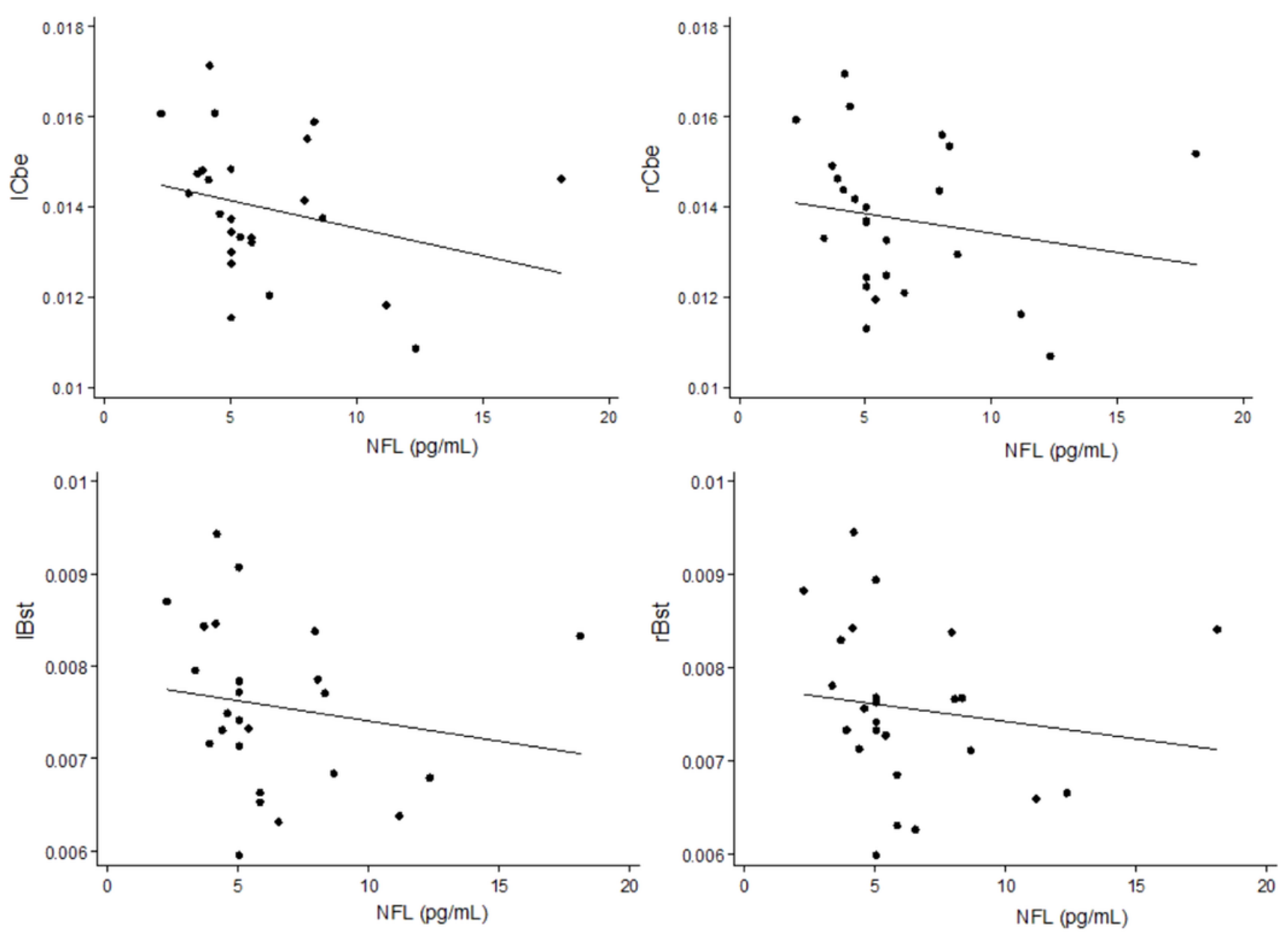

*ICbe: left cerebellum; rCbe: right cerebellum; IBst: left brainstem; rBst: right Brainstem

\section{Figure 2}

Spearman correlations for cerebellum and brainstem volumetric measures and $\mathrm{pNfL}$ concentrations in HIV-infected patients

\section{Supplementary Files}

This is a list of supplementary files associated with this preprint. Click to download.

- Supplementarymaterial1.docx 